\title{
Body Waves in Fractured Porous Media Saturated by Two Immiscible Newtonian Fluids
}

\author{
KAGAN TUNCAY* and M. YAVUZ CORAPCIOGLU \\ Department of Civil Engineering, Texas A\&M University, College Station, TX 77840-3136, U.S.A.
}

(Received: 6 March 1995; in final form: 18 December 1995)

\begin{abstract}
A study of body waves in fractured porous media saturated by two fluids is presented. We show the existence of four compressional and one rotational waves. The first and third compressional waves are analogous to the fast and slow compressional waves in Biot's theory. The second compressional wave arises because of fractures, whereas the fourth compressional wave is associated with the pressure difference between the fluid phases in the porous blocks. The effects of fractures on the phase velocity and attenuation coefficient of body waves are numerically investigated for a fractured sandstone saturated by air and water phases. All compressional waves except the first compressional wave are diffusive-type waves, i.e., highly attenuated and do not exist at low frequencies.
\end{abstract}

Key words: body waves, fracture porous media, Biot's theory, immiscible Newtonian fluids, compressional waves

\section{Introduction}

Biot's work on wave propagation in saturated porous media dominated the field over three decades and influenced the direction of future research more than any other person who ever worked in this area (Biot, 1956a,b). Biot's theory predicts two compressional waves and one rotational wave in a saturated porous medium. The first compressional wave is analogous to the compressional wave in elastic solids. The second compressional wave is slower and highly attenuated. Because of its highly dissipative behavior, it took more than 20 years to observe the second compressional wave (Plona, 1980).

Brutsaert (1964) extended Biot's theory and predicted three compressional waves in elastic porous media saturated by two fluids. The third compressional wave was also predicted by Garg and Nayfeh (1986), Santos et al. (1990a,b), and Tuncay and Corapcioglu (1996, 1996a) employed the volume averaging technique to derive the macroscopic constitutive relations as well as the macroscopic mass and momentum balance equations. Their analysis showed that the third compressional wave is associated with the pressure difference of the fluid phases. The third compressional wave has the lowest velocity and it is highly attenuated like the second compressional wave. Wave propagation in saturated porous media has been

* Now at Izmir Institute of Technology, Faculty of Engineering, Gaziosmanpasa Bulvari, No.16, Cankaya, Izmir, Turkey. 
extensively studied, e.g., Bedford and Stern (1983), Nakoryakov et al. (1989), Bear and Corapcioglu (1989). A review of the subject has been presented in Corapcioglu (1991).

Deformable fractured porous media have been the subject of very few studies. Aifantis and co-workers published a series of papers on consolidation of saturated fractured porous media (Wilson and Aifantis, 1982; Beskos and Aifantis, 1986; Khaled et al., 1986). The phenomenological coefficients of Aifantis' theory were expressed in terms of measurable coefficients by Wilson and Aifantis (1982). Uniqueness and general solutions were investigated by Beskos and Aifantis (1986). Wilson and Aifantis (1984) extended Aifantis' work to study wave propagation in saturated fractured porous media and predicted three compressional waves. The third compressional wave arises because of the fluid phase in the fractures (secondary pores). Similar results were obtained by Beskos who published a series of papers on the dynamics of fractured porous media (Beskos, 1989; Beskos et al., 1989a,b).

In this study, we investigate the types and characteristics of waves in infinite isotropic fractured porous media saturated by two fluids based on the governing equations derived by Tuncay and Corapcioglu (1996). We present a quantitative investigation of body waves for a fractured sandstone saturated by air and water phases.

\section{Final Set of Governing Equations}

We start by introducing the constitutive relations and governing equations for fractured porous media saturated by two fluids obtained by Tuncay and Corapcioglu (1996). In the derivation, the fluid phases are assumed to be immiscible and at rest. Furthermore, the solid phase is assumed to be isotropic, experiencing small deformations and providing all shear resistance of the porous medium. Momentum transfer terms are expressed in terms of intrinsic and relative permeabilities assuming the validity of Darcy's law in fractured porous media. The theory is limited to low frequency wave propagation. The medium is assumed to have fractures which are referred as secondary pores. The pores of the nonfractured part of the porous medium are referred as primary pores. The secondary pores are saturated by wetting fluid whereas the primary pores are saturated by wetting and nonwetting fluids. The mass transfer between the primary pores and secondary pores per unit volume is approximated by Tuncay and Corapcioglu (1996)

$$
\begin{aligned}
& \frac{1}{V} \int_{S_{2 f}} \rho_{2}\left(u-v_{2}\right) \cdot n_{2} \mathrm{~d} A \\
& \quad=-\frac{1}{V} \int_{S_{f 2}} \rho_{2}\left(u-v_{f}\right) \cdot n_{f} \mathrm{~d} A=R \rho_{2}\left(\bar{P}_{f}-\bar{P}_{2}\right),
\end{aligned}
$$


where $R$ is a material property of the porous medium and the wetting fluid, $\bar{P}_{2}$ and $\bar{P}_{f}$ are the pressures in fluid phase 2 and phase $f$, respectively. From now on, subscripts $s, 1,2$ and $f$ will refer to the solid phase, nonwetting phase, wetting phase in the primary pores, and the fluid phase in the fractures, respectively. Barenblatt et al. (1960) proposed $R$ as

$$
R=\frac{c K_{f} A^{2}}{\mu_{2}},
$$

where $A$ is the area of fracture-block contact per unit volume, $c$ denotes a dimensionless shape factor of the fractured medium and $K_{f}$ is the permeability of the fractures. The macroscopic constitutive relations are obtained as

$$
\begin{aligned}
\alpha_{s} \bar{\tau}_{s}= & \left(a_{11} \nabla \cdot \bar{u}_{s}+a_{12} \nabla \cdot \bar{u}_{1}+a_{13} \nabla \cdot \bar{u}_{2}+a_{14} \nabla \cdot \bar{u}_{f}+\right. \\
& \left.+\left(\frac{a_{14}}{\alpha_{f}}-\frac{a_{13}}{\alpha_{2}}\right) M\right) \cdot I+ \\
& +G_{f r}\left(\nabla \bar{u}_{s}+\left(\nabla \bar{u}_{s}\right)^{T}-\frac{2}{3} \nabla \cdot \bar{u}_{s}\right), \\
\alpha_{1} \bar{P}_{1}= & a_{21} \nabla \cdot \bar{u}_{s}+a_{22} \nabla \cdot \bar{u}_{1}+a_{23} \nabla \cdot \bar{u}_{2}+ \\
& +a_{24} \nabla \cdot \bar{u}_{f}+\left(\frac{a_{24}}{\alpha_{f}}-\frac{a_{23}}{\alpha_{2}}\right) M, \\
\alpha_{2} \bar{P}_{2}= & a_{31} \nabla \cdot \bar{u}_{s}+a_{32} \nabla \cdot \bar{u}_{1}+a_{33} \nabla \cdot \bar{u}_{2}+ \\
& +a_{34} \nabla \cdot \bar{u}_{f}+\left(\frac{a_{34}}{\alpha_{f}}-\frac{a_{33}}{\alpha_{2}}\right) M, \\
\alpha_{f} \bar{P}_{f}= & a_{41} \nabla \cdot \bar{u}_{s}+a_{42} \nabla \cdot \bar{u}_{1}+a_{43} \nabla \cdot \bar{u}_{2}+ \\
& +a_{44} \nabla \cdot \bar{u}_{f}+\left(\frac{a_{44}}{\alpha_{f}}-\frac{a_{43}}{\alpha_{2}}\right) M .
\end{aligned}
$$

The reader is referred to Tuncay and Corapcioglu (1996) for expressions of $a_{i j}$ in terms of material parameters: $K_{s}$, bulk modulus of the solid grains; $K_{f r}$, bulk modulus of the fractured porous medium; $K_{f r}^{m}$, bulk modulus of the nonfractured 
porous medium; $\alpha_{i}$, volume fraction of phase $i ; \mathrm{S}_{1}$, saturation of the nonwetting fluid phase; $K_{1}$, bulk modulus of the nonwetting fluid phase; $K_{2}$, bulk modulus of the wetting fluid phase; $P_{\text {cap }}^{\prime}$, derivative of the capillary pressure-saturation relation with respect to $S_{1} ; G_{f r}$, shear modulus of the solid matrix and $F$, a material propertiy associated with the changes in volume fraction of fractures. $M$ is given by

$$
\frac{\partial M}{\partial t}=R\left(\bar{P}_{f}-\bar{P}_{2}\right) .
$$

We note that $\rho_{2} \partial M / \partial t$ is equal to the mass transfer rate of wetting fluid phase between the primary pores and fractures. The volume averaged momentum balance equations are obtained as

$$
\begin{aligned}
\left\langle\rho_{s}\right\rangle \frac{\partial^{2} \bar{u}_{s}}{\partial t^{2}}= & \nabla\left(\left(a_{11}+\frac{G_{f r}}{3}\right) \nabla \cdot \bar{u}_{s}+a_{12} \nabla \cdot \bar{u}_{1}+a_{13} \nabla \cdot \bar{u}_{2}+\right. \\
& \left.+a_{14} \nabla \cdot \bar{u}_{f}+\left(\frac{a_{14}}{\alpha_{f}}-\frac{a_{13}}{\alpha_{2}}\right) M\right)+\nabla \cdot\left(G_{f r} \nabla \bar{u}_{s}\right)+ \\
& +C_{1}\left(\bar{v}_{1}-\bar{v}_{s}\right)+C_{2}\left(\bar{v}_{2}-\bar{v}_{s}\right)+C_{3}\left(\bar{v}_{f}-\bar{v}_{s}\right) \\
\left\langle\rho_{1}\right\rangle \frac{\partial^{2} \bar{u}_{1}}{\partial t^{2}}= & \nabla\left(a_{21} \nabla \cdot \bar{u}_{s}+a_{22} \nabla \cdot \bar{u}_{1}+a_{23} \nabla \cdot \bar{u}_{2}+\right. \\
& \left.+a_{24} \nabla \cdot \bar{u}_{f}+\left(\frac{a_{24}}{\alpha_{f}}-\frac{a_{23}}{\alpha_{2}}\right) M\right)-C_{1}\left(\bar{v}_{1}-\bar{v}_{s}\right), \\
\left\langle\rho_{2}\right\rangle \frac{\partial^{2} \bar{u}_{2}}{\partial t^{2}}= & \nabla\left(a_{31} \nabla \cdot \bar{u}_{s}+a_{32} \nabla \cdot \bar{u}_{1}+a_{33} \nabla \cdot \bar{u}_{2}+\right. \\
& \left.+a_{34} \nabla \cdot \bar{u}_{f}+\left(\frac{a_{34}}{\alpha_{f}}-\frac{a_{33}}{\alpha_{2}}\right) M\right)-C_{2}\left(\bar{v}_{2}-\bar{v}_{s}\right) \\
\left\langle\rho_{f}\right\rangle \frac{\partial^{2} \bar{u}_{f}}{\partial t^{2}}= & \nabla\left(a_{41} \nabla \cdot \bar{u}_{s}+a_{42} \nabla \cdot \bar{u}_{1}+a_{43} \nabla \cdot \bar{u}_{2}+\right. \\
& \left.+a_{44} \nabla \cdot \bar{u}_{f}+\left(\frac{a_{44}}{\alpha_{f}}-\frac{a_{43}}{\alpha_{2}}\right) M\right)-C_{3}\left(\bar{v}_{f}-\bar{v}_{s}\right)
\end{aligned}
$$

where

$$
C_{1}=\frac{\left(1-\alpha_{s}-\alpha_{f}\right)^{2} S_{1}^{2} \mu_{1}}{K_{p} k_{r 1}}
$$




$$
\begin{aligned}
& C_{2}=\frac{\left(1-\alpha_{s}-\alpha_{f}\right)^{2}\left(1-S_{1}\right)^{2} \mu_{2}}{K_{p} k_{r 2}}, \\
& C_{3}=\frac{\alpha_{f}^{2} \mu_{2}}{K_{f}} .
\end{aligned}
$$

In Equations (13)-(14), $K_{p}$ is the intrinsic permeability of the nonfractured porous medium and $k_{r i}$ is the relative permeability of phase $i, K_{f}$ is the intrinsic permeability of the fractures and $\mu_{i}$ is the viscosity of phase $i$. Equations (5)-(11) form the final set of 15 equations with 15 unknowns $\bar{u}_{s}, \bar{u}_{1}, \vec{u}_{2}, \bar{u}_{f}, \bar{P}_{f}, \bar{P}_{2}$ and $M$.

\section{Compressional Waves}

To investigate compressional waves, we apply divergence to Equations (8)-(11) to obtain

$$
\begin{aligned}
\left\langle\rho_{s}\right\rangle \frac{\partial^{2} \varepsilon_{s}}{\partial t^{2}}= & a_{11}^{*} \nabla^{2} \varepsilon_{s}+a_{12} \nabla^{2} \varepsilon_{1}+a_{13} \nabla^{2} \varepsilon_{2}+a_{14} \nabla^{2} \varepsilon_{f}+ \\
& +\left(\frac{a_{14}}{\alpha_{f}}-\frac{a_{13}}{\alpha_{2}}\right) \nabla^{2} M+C_{1}\left(\frac{\partial \varepsilon_{1}}{\partial t}-\frac{\partial \varepsilon_{s}}{\partial t}\right)+C_{2}\left(\frac{\partial \varepsilon_{2}}{\partial t}\right)+ \\
& +C_{3}\left(\frac{\partial \varepsilon_{f}}{\partial t}-\frac{\partial \varepsilon_{s}}{\partial t}\right) \\
\left\langle\rho_{1}\right\rangle \frac{\partial^{2} \varepsilon_{1}}{\partial t^{2}}= & a_{21} \nabla^{2} \varepsilon_{s}+a_{22} \nabla^{2} \varepsilon_{1}+a_{23} \nabla^{2} \varepsilon_{2}+a_{24} \nabla^{2} \varepsilon_{f}+ \\
& +\left(\frac{a_{24}}{\alpha_{f}}-\frac{a_{23}}{\alpha_{2}}\right) \nabla^{2} M-C_{1}\left(\frac{\partial \varepsilon_{1}}{\partial t}-\frac{\partial \varepsilon_{s}}{\partial t}\right) \\
\left\langle\rho_{2}\right\rangle \frac{\partial^{2} \varepsilon_{2}}{\partial t^{2}}= & a_{31} \nabla^{2} \varepsilon_{s}+a_{32} \nabla^{2} \varepsilon_{1}+a_{33} \nabla^{2} \varepsilon_{2}+a_{34} \nabla^{2} \varepsilon_{f} \\
& +\left(\frac{a_{34}}{\alpha_{f}}-\frac{a_{33}}{\alpha_{2}}\right) \nabla^{2} M-C_{2}\left(\frac{\partial \varepsilon_{2}}{\partial t}-\frac{\partial \varepsilon_{s}}{\partial t}\right) \\
& +\left(\frac{a_{44}}{\alpha_{f}}-\frac{a_{43}}{\alpha_{2}}\right) \nabla^{2} M-C_{3}\left(\frac{\partial \varepsilon_{f}}{\partial t}-\frac{\partial \varepsilon_{s}}{\partial t}\right) \\
\left\langle\rho_{f}\right\rangle \frac{\partial^{2} \varepsilon_{f}}{\partial t^{2}}= & a_{41} \nabla^{2} \varepsilon_{s}+a_{42} \nabla^{2} \varepsilon_{1}+a_{43} \nabla^{2} \varepsilon_{2}+a_{44} \nabla^{2} \varepsilon_{f}+ \\
&
\end{aligned}
$$


where $\varepsilon_{j}=\nabla \cdot \bar{u}_{j}$ and $a_{11}^{*}=a_{11}+4 G_{f r} / 3$. We state the dilatational plane harmonic waves propagating along the $z$ direction by

$$
\begin{aligned}
& \varepsilon_{j}=B_{j} \mathrm{e}^{i(\xi z-\omega t)}, \quad M=B_{m} \mathrm{e}^{i(\xi z-\omega t)}, \\
& P_{f}=A_{j} \mathrm{e}^{i(\xi z-\omega t)}, \quad P_{p}=A_{p} \mathrm{e}^{i(\xi z-\omega t)},
\end{aligned}
$$

where $B_{j}, B_{m}, A_{f}$ and $A_{p}$ are the wave amplitudes, $\xi$ is the wave number, $\omega$ is the frequency and $i$ is the imaginary number. The phase velocity is defined as $c=\omega / \xi_{\text {r }}$ where $\xi_{r}$ is the real part of the wave number. The imaginary part of $\xi$ is called the attenuation coefficient. Substitution of Equation (19) in Equations (5)-(11) yields a set of homogeneous algebraic equations and for nontrivial solution the determinant of the coefficient matrix must be equal to zero. The coefficient matrix is obtained as shown in Equation (20) opposite.

For a given $\omega$, the determinant of the coefficient matrix equated to zero, is known as the 'dispersion equation' in wave mechanics literature. It is an eighthorder polynomial in terms of wave number. The polynomial contains only the even powers of the wave number. Because the amplitude of the waves should decrease as they propagate, imaginary part of the wave number must be greater than zero. This implies the existence of four compressional waves. Since the determinant of the coefficient matrix is extremely lengthy, the reader is referred to Tuncay (1995) for the explicit expression of the determinant. When $M=0$, i.e., no mass exchange between the porous blocks and fractures, the number of unknowns reduce to twelve $\left(\bar{u}_{g}, \bar{u}_{1}, \bar{u}_{2}\right.$ and $\left.\bar{u}_{f}\right)$ and the coefficient matrix reduces to a four by four matrix.

\section{Rotational Waves}

To investigate the rotational waves, we apply curl operator to Equations (8)-(11)

$$
\begin{aligned}
\left\langle\rho_{s}\right\rangle \frac{\partial^{2} \Omega_{s}}{\partial t^{2}}= & G_{f r} \nabla^{2} \Omega_{s}+C_{1}\left(\frac{\partial \Omega_{1}}{\partial t}-\frac{\partial \Omega_{s}}{\partial t}\right)+C_{2}\left(\frac{\partial \Omega_{2}}{\partial t}-\frac{\partial \Omega_{s}}{\partial t}\right)+ \\
& +C_{3}\left(\frac{\partial \Omega_{f}}{\partial t}-\frac{\partial \Omega_{s}}{\partial t}\right), \\
\left\langle\rho_{1}\right\rangle \frac{\partial^{2} \Omega_{1}}{\partial t^{2}}= & -C_{1}\left(\frac{\partial \Omega_{1}}{\partial t}-\frac{\partial \Omega_{s}}{\partial t}\right), \\
\left\langle\rho_{2}\right\rangle \frac{\partial^{2} \Omega_{2}}{\partial t^{2}}= & -C_{2}\left(\frac{\partial \Omega_{2}}{\partial t}-\frac{\partial \Omega_{s}}{\partial t}\right), \\
\left\langle\rho_{f}\right\rangle \frac{\partial^{2} \Omega_{f}}{\partial t^{2}}= & -C_{3}\left(\frac{\partial \Omega_{f}}{\partial t}-\frac{\partial \Omega_{s}}{\partial t}\right),
\end{aligned}
$$

where $\Omega_{j}=\nabla \times \bar{u}_{j}$. Substitution of harmonic waves in (21)-(24) yields 


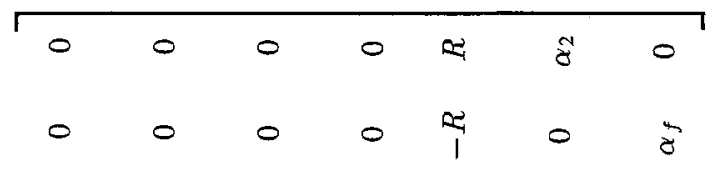

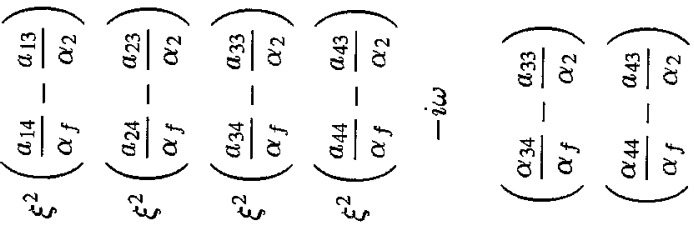

बํํ

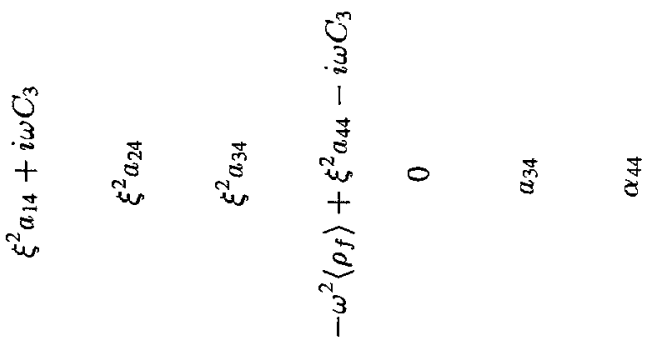

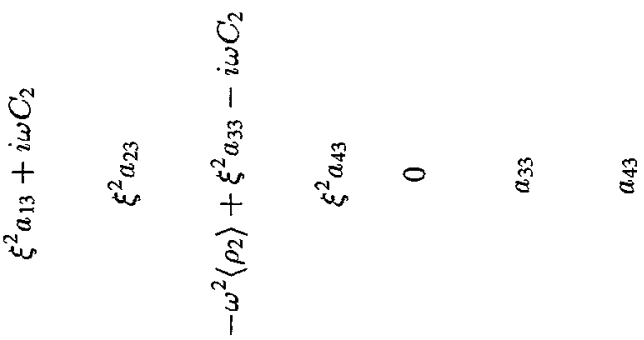

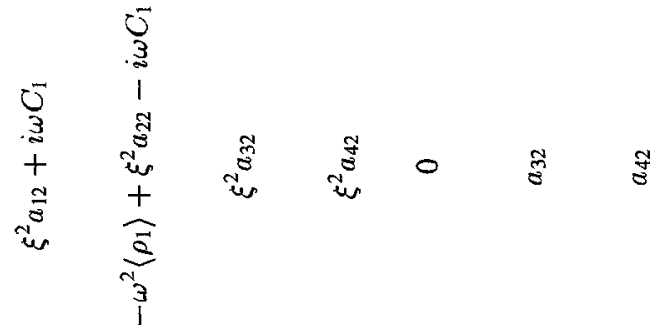

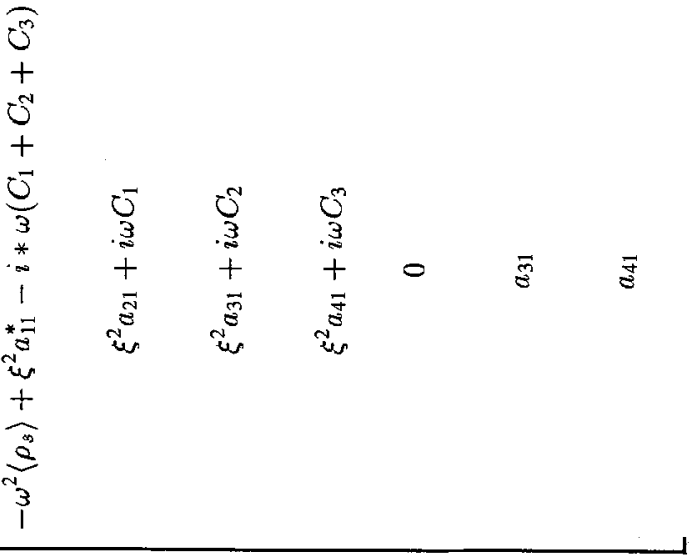




$$
\begin{aligned}
& \left\{-\omega^{2}\left[\begin{array}{cccc}
\left\langle\rho_{s}\right\rangle & 0 & 0 & 0 \\
0 & \left\langle\rho_{1}\right\rangle & 0 & 0 \\
0 & 0 & \left\langle\rho_{2}\right\rangle & 0 \\
0 & 0 & 0 & \left\langle\rho_{f}\right\rangle
\end{array}\right]+\xi^{2}\left[\begin{array}{cccc}
G_{f r} & 0 & 0 & 0 \\
0 & 0 & 0 & 0 \\
0 & 0 & 0 & 0 \\
0 & 0 & 0 & 0
\end{array}\right]+\right. \\
& \left.+i \omega\left[\begin{array}{cccc}
-C_{1}-C_{2}-C_{3} & C_{1} & C_{2} & C_{3} \\
C_{1} & -C_{1} & 0 & 0 \\
C_{2} & 0 & -C_{2} & 0 \\
C_{3} & 0 & 0 & -C_{3}
\end{array}\right]\right\}\left[\begin{array}{c}
B_{s} \\
B_{1} \\
B_{2} \\
B_{f}
\end{array}\right]=\left[\begin{array}{l}
0 \\
0 \\
0 \\
0
\end{array}\right]
\end{aligned}
$$

Similar to compressional waves, the dispersion equation is the determinant of the coefficient matrix (Tuncay, 1995). The determinant is a second-order polynomial containing only even powers of the wave number which implies the existence of one rotational wave in a fractured porous medium.

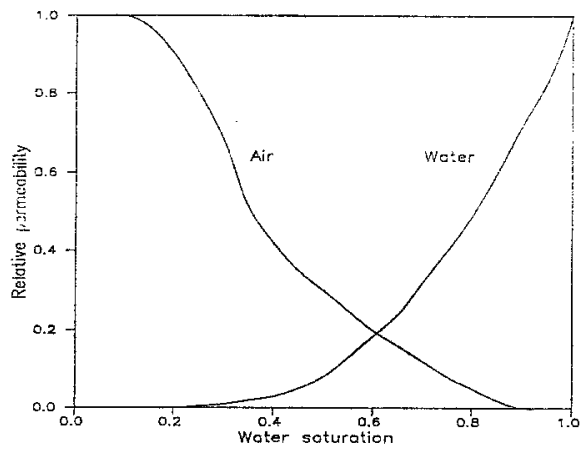

Figure 1. Relative permeability curves of a sandstone saturated by air and water phase (after Wyckoff and Botset, 1936).

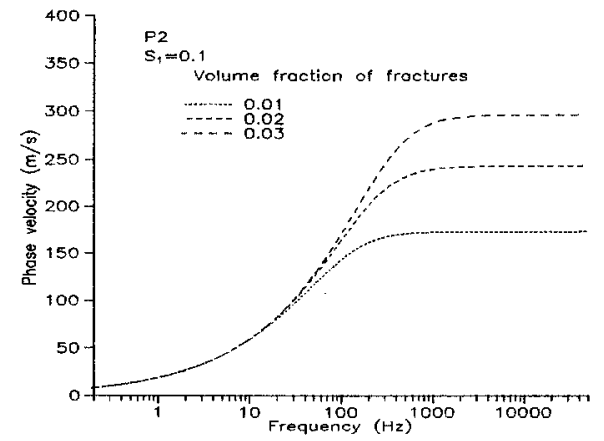

Figure 3. Frequency dependence of the phase velocity of P2.

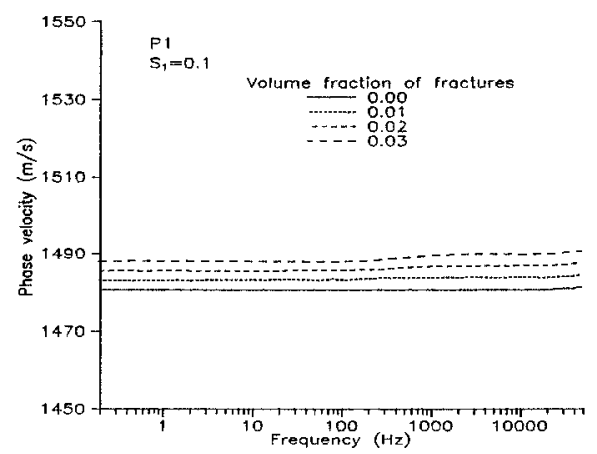

Figure 2. Frequency dependence of the phase velocity of $\mathrm{P} 1$.

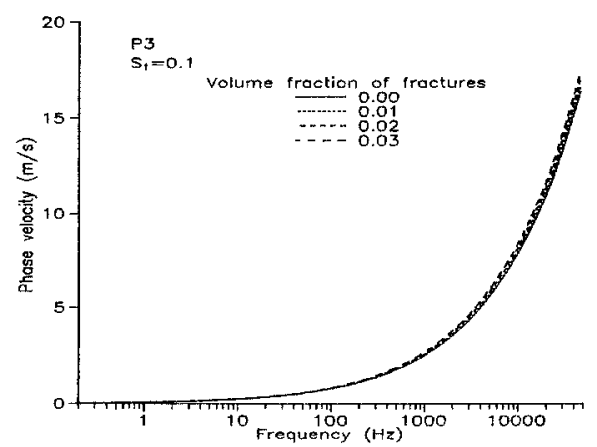

Figure 4. Frequency dependence of the phase velocity of $\mathrm{P3}$. 
Table I. Material parameters of a fractured sandstone saturated by air and water phases.

\begin{tabular}{lll}
\hline Parameter & Symbol & Value \\
\hline Bulk modulus of fractured medium & $K_{f r}$ & $1.44 \mathrm{GPa}$ \\
Bulk modulus of nonfractured blocks & $K_{f r}^{m}$ & $3.00 \mathrm{GPa}$ \\
Bulk modulus of solid grains & $K_{s}$ & $35.00 \mathrm{GPa}$ \\
Shear modulus of solid matrix & $G_{f r}$ & $1.02 \mathrm{GPa}$ \\
Density of solid grains & $\rho_{s}$ & $2650 \mathrm{~kg} / \mathrm{m}^{3}$ \\
Intrinsic permeability of fractures & $K_{f}$ & $10^{-11} \mathrm{~m}^{2}$ \\
Intrinsic permeability of nonfractured medium & $K_{p}$ & $10^{-13} \mathrm{~m}^{2}$ \\
Volume fraction of solid phase & $\alpha_{s}$ & 0.77 \\
Density of water & $\rho_{2}$ & $997 \mathrm{~kg} / \mathrm{m}^{3}$ \\
Bulk modulus of water & $K_{2}$ & $2.25 \mathrm{GPa}$ \\
Viscosity of water & $\mu_{2}$ & $10^{-3} \mathrm{~Pa} . \mathrm{s}$ \\
Density of air & $\rho_{1}$ & $1.1 \mathrm{~kg} / \mathrm{m}^{3}$ \\
Bulk modulus of air & $K_{1}$ & $0.145 \mathrm{MPa}$ \\
Viscosity of air & $\mu_{1}$ & $1.8 \times 10^{-6} \mathrm{~Pa} . \mathrm{s}$ \\
Rate coefficient of mass transfer & $R$ & $2 \times 10^{-16} 1 / \mathrm{Pa} . \mathrm{s}$ \\
A dimensionless material parameter & $F$ & 0.8 \\
\hline
\end{tabular}

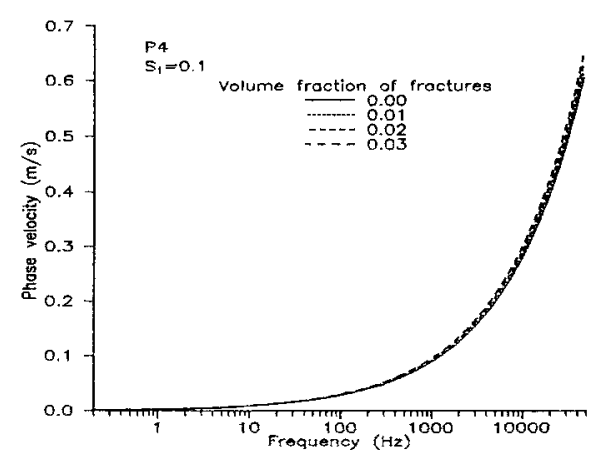

Figure 5. Frequency dependence of the phase velocity of $\mathrm{P4}$.

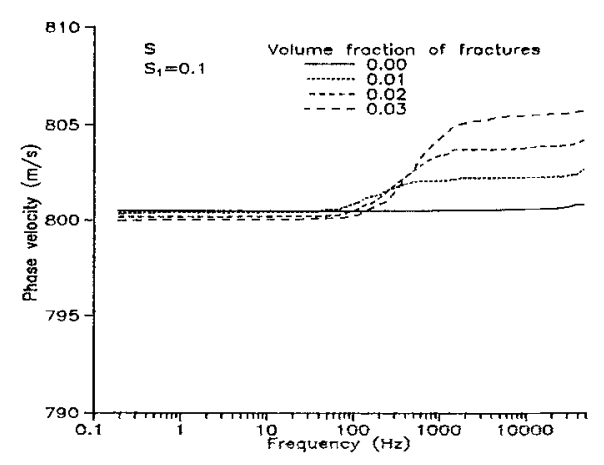

Figure 6. Frequency dependence of the phase velocity of $\mathrm{S}$.

\section{Numerical Results}

The dispersion equations for compressional and rotational waves are the determinants of Equations (20) and (25) equated to zero, respectively. For a given frequency, these equations are solved for the wave number. The phase velocity is defined as $c=\omega / \xi_{r}$, where $\xi_{r}$ is the real part of the wave number. The imaginary part of $\xi$ is called the attenuation coefficient. The material parameters in Table I correspond to a fractured sandstone saturated by air and water phases. Van Genuchten's 
(1980) closed form expressions for the capillary pressure-saturation relations are employed to obtain $P_{\text {cap }}^{\prime}$ which appear in $a_{i j}$ expressions. Van Genuchten proposed that

$$
\frac{S_{2}-S_{r 2}}{S_{m 2}-S_{r 2}}=\left(1+\left(\frac{\alpha P_{\text {cap }}}{100}\right)^{n}\right)^{-m}
$$

where $P_{\text {cap }}$ is the capillary pressure $\left(\mathrm{N} / \mathrm{m}^{2}\right), S_{2}$ is the water saturation, $S_{m 2}$ is the upper limit of water saturation, $S_{r 2}$ is the irreducible water saturation, $m=1-1 / n$, and $\alpha$ and $n$ are material parameters. Realizable parameters for a sandstone are $\alpha=0.025, n=10$ and $\mathrm{S}_{r 2}=0.0$. We use the relative permeability curves of Wyckoff and Botset (1936) for a sandstone shown in Figure 1.

Figures 2-6 illustrate the frequency dependence of body waves for different values of volume fraction of fractures $\alpha_{f}$ when the air saturation $S_{1}=0.1$. From now on, we will represent the compressional waves by 'P' and rotational wave by ' $S$ '. Since there are four compressional waves, we will number them according to the magnitude of their phase velocity, P1 being the fastest. As seen in Figure 2, as the volume fraction of the fractures increases, the phase velocity of P1 increases. However, the increase is not very significant. Comparing Figures 2-5, we conclude that the volume fraction of fractures causes a notable change in the phase velocity of $\mathrm{P} 2$ only. Especially, the phase velocities of $\mathrm{P} 3$ and $\mathrm{P} 4$ appear to be independent of the volume fraction of fractures. We should note that $\mathrm{P} 2$ vanishes when as the volume fraction of fractures approaches zero. We conclude that $\mathrm{P} 2$ is associated with the fluid phase in the fractures and vanishes when the medium is nonfractured. As seen in Figure 6, the rotational wave $S$ has the second highest phase velocity.

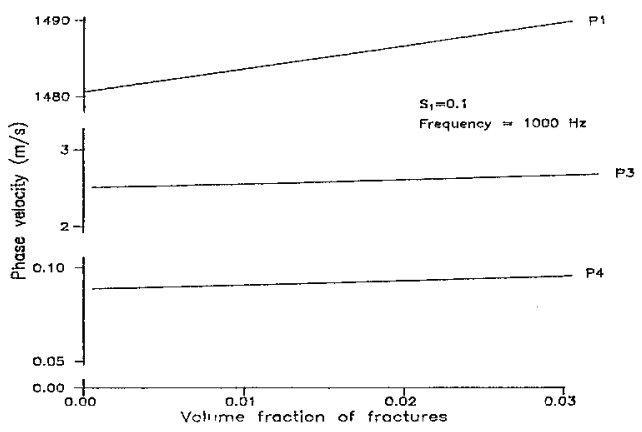

Figure 7. Effect of volume of fractures on the velocity of $\mathrm{P} 1, \mathrm{P} 3$, and $\mathrm{P} 4$.

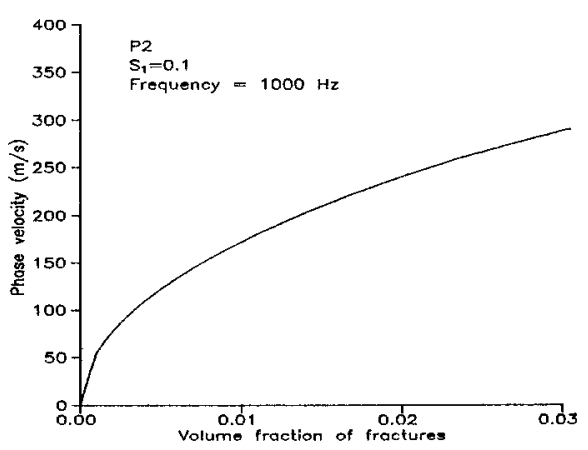

Figure 8. Effect of volume of fractures on the phase velocity of $\mathrm{P} 2$. 


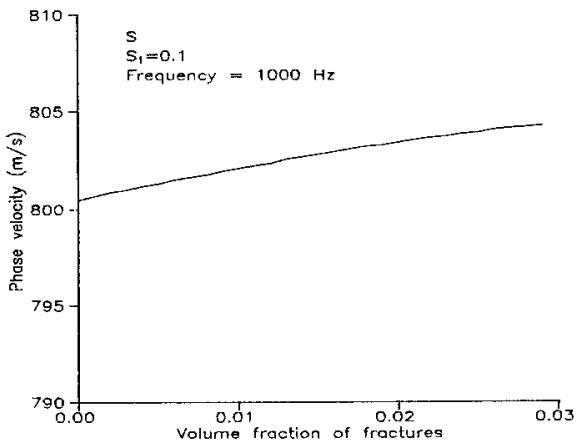

Figure 9. Effect of volume of fractures on the phase velocity of $S$.

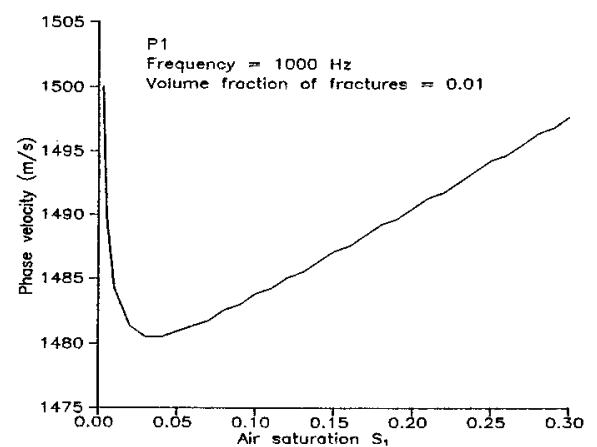

Figure 10. Effect of air saturation on the phase velocity of P1.

The effect of volume fraction of fractures on the phase velocities is shown in Figures 7-9. The air saturation and frequency are taken as 0.1 and $1000 \mathrm{~Hz}$, respectively. We observe almost a linear variation in the phase velocities of P1 and $S$ as the volume fraction of fractures changes in Figures 7 and 9, respectively. Since the reported volume fractions of fractures in the literature are normally less than 0.03 , the horizontal scale of Figures 7-9 is limited to that value. We find that even when $\alpha_{f}=0.03$, the phase velocity of P1 increases less than $1 \%$. The phase velocity of $\mathrm{P} 2$ starts from zero and rises as $\alpha_{f}$ increases (see Figure 8). P3 and P4 show no dependence on the volume fraction of fractures.

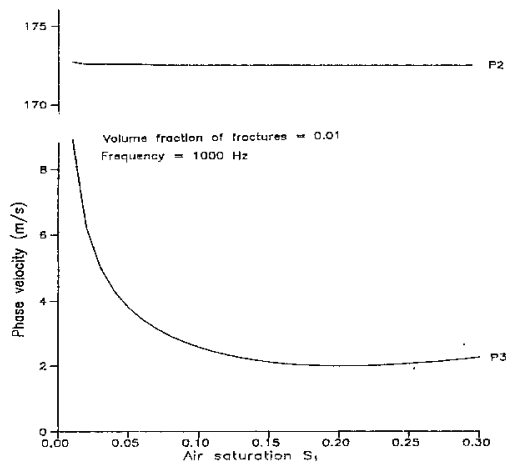

Figure 11. Effect of air saturation on the phase velocity of $\mathrm{P} 2$ and $\mathrm{P} 3$.

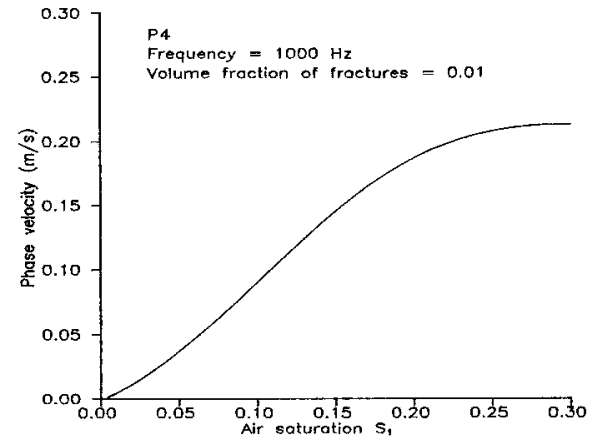

Figure 12. Effect of air saturation on the phase velocity of $\mathrm{P} 4$.

Then, we investigate the saturation dependence of phase velocities (Figures 1013). The volume fraction of fractures and frequency are taken as 0.01 and $1000 \mathrm{~Hz}$, respectively. In Figure 10, we observe a significant drop in the phase velocity of $\mathrm{P} 1$. This is due to the high compressibility of the air phase. This behavior was observed in several experimental studies (Domenico, 1974; Murphy, 1982). After a minimum value, the phase velocity of $\mathrm{P} 1$ increases with increasing air saturation. 
The phase velocity of $\mathrm{P} 2$ does not change with changes in the air saturation (Figure 11). The phase velocity of $P 3$ behaves similar to that of P1. As seen in Figure 12 , P4 vanishes when the pores are fully saturated by water. We conclude that P4 arises because of the second fluid phase in the primary pores. In nonfractured porous media, Tuncay and Corapcioglu (1995) showed that this compressional wave depends on the capillary-pressure-saturation relation and vanishes when the pressures of the fluid phases are equal. Figure 13 illustrates the dependence of phase velocity of $S$ on the air saturation. We observe almost a linear variation as the air saturation increases.

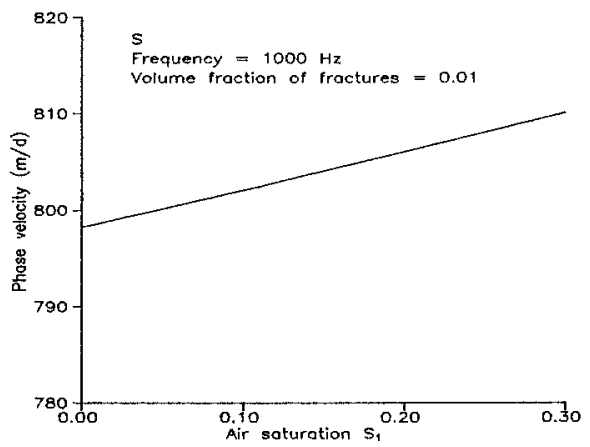

Figure 13. Effect of air saturation on the phase velocity of $\mathrm{S}$.

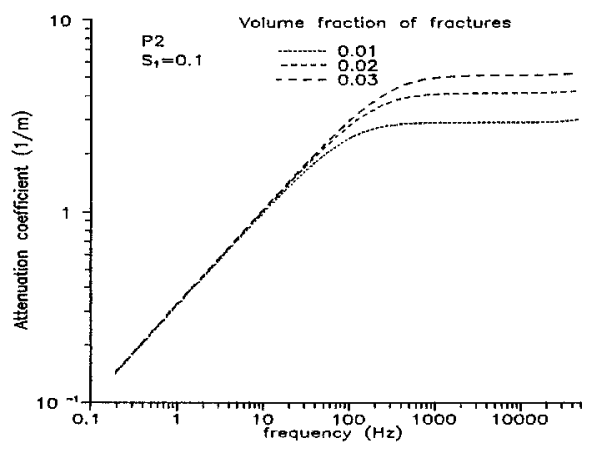

Figure 15. Frequency dependence of the attenuation coefficient of $\mathrm{P} 2$.

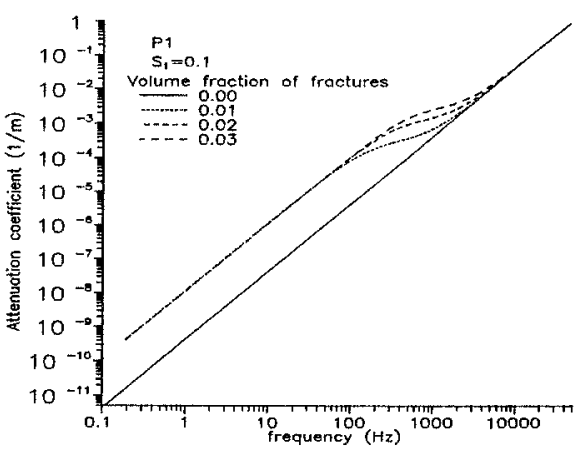

Figure 14. Frequency dependence of the attenuation coefficient of $\mathrm{P} 1$.

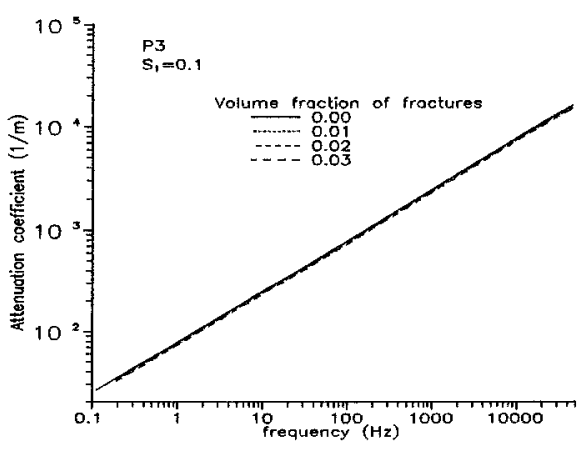

Figure 16. Frequency dependence of the attenuation coefficient of $\mathrm{P} 3$. 


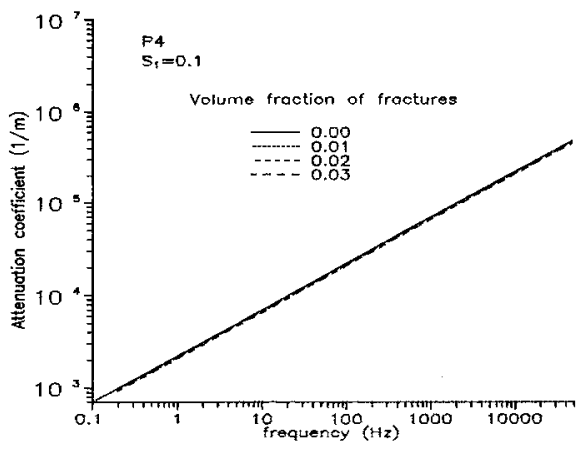

Figure 17. Frequency dependence of the attenuation coefficient of P4.

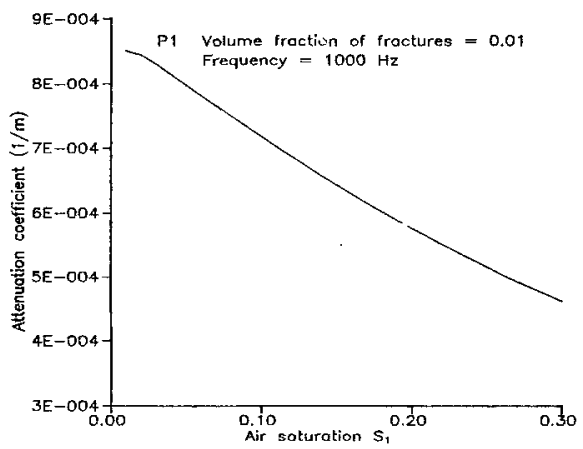

Figure 19. Effect of air saturation on the attenuation coefficient of $\mathbf{P 1}$.

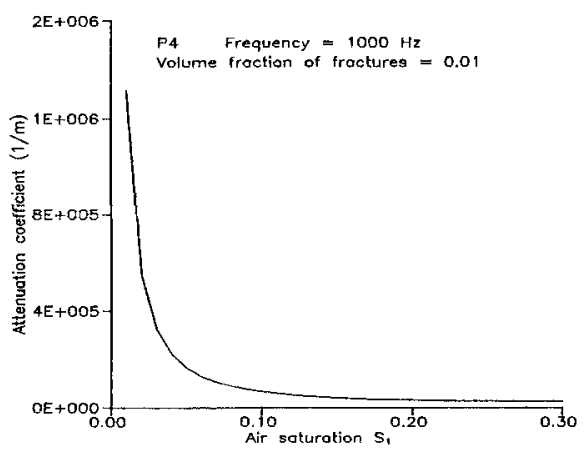

Figure 21. Effect of air saturation on the attenuation coefficient of $\mathrm{P} 4$.

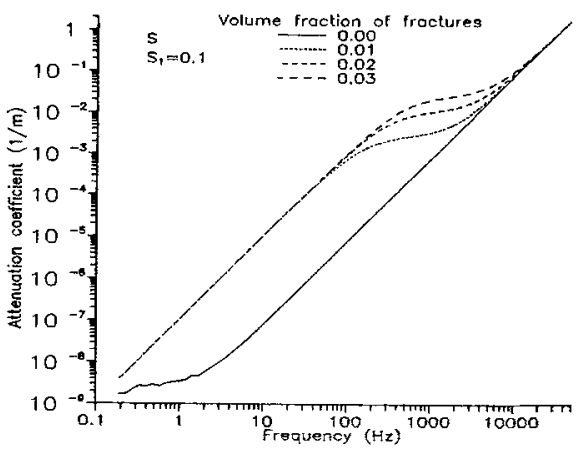

Figure 18. Frequency dependence of the attenuation coefficient of $S$.

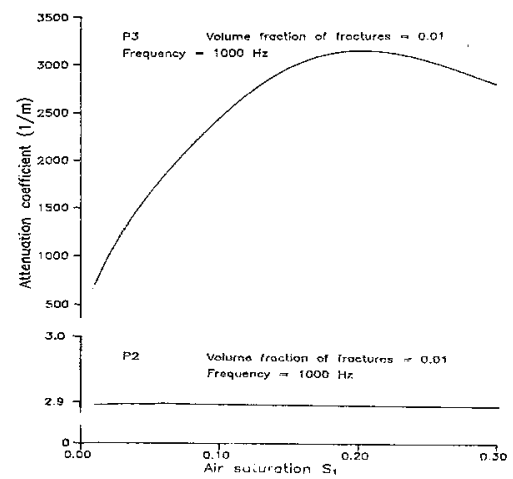

Figure 20. Effect of air saturation on the attenuation coefficient of $\mathrm{P} 2$ and $\mathrm{P} 3$.

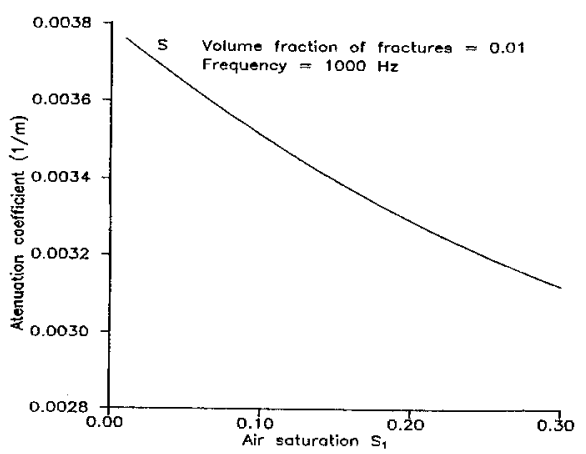

Figure 22. Effect of air saturation on the attenuation coefficient of $S$.

Figures 14-18 illustrate the frequency dependence of the attenuation coefficients. The air saturation $S_{1}$ is taken as 0.1 . The volume fraction of fractures changes the order of magnitude of the attenuation coefficient of P1 and S more than one order for frequencies less that $1000 \mathrm{~Hz}$. This can be explained by the 
high intrinsic permeability of the fractures. The attenuation coefficient of $\mathrm{P} 2$ shows dependence on the volume fraction of fractures for frequencies higher than $100 \mathrm{~Hz}$ (see Figure 15). The attenuation coefficient of $\mathrm{P} 2$ increases with the volume fraction of fractures. As seen in Figures 16-17, the attenuation coefficients of P3 and P4 do not depend on the volume fraction of fractures. As in P1, attenuation coefficient of $S$ significantly depends on the volume fraction of fractures (see Figure 18).

Figures 19-22 show the attenuation coefficients of the body waves as a function of the air saturation $S_{1}$. The volume fraction fractures and frequency are taken as 0.01 and $1000 \mathrm{~Hz}$, respectively. The attenuation coefficients of P1 and $S$ decrease as air saturation $S_{1}$ increases (see Figures 19 and 22). The attenuation coefficient of P2 is almost independent of the saturation. As seen in Figures 20 and 21, the attenuation coefficients of $\mathrm{P} 3$ and $\mathrm{P} 4$ significantly depend on the saturation. As noted earlier $\mathrm{P} 4$ disappears when there is only one fluid phase in the primary pores. As seen in Figure 21, the attenuation coefficient of P4 reaches to very large values when the air saturation approaches zero.

\section{Conclusions}

We studied the characteristics of body waves in fractured porous media saturated by two fluids. We showed the existence of four compressional and one rotational waves. The fastest wave (P1) is analogous to Biot's fast wave. The second wave (P2) arises because of the fluid phase in the fractures and it vanishes when the medium is not fractured. The third compressional wave (P3) corresponds to the slow wave of Biot's theory. The fourth compressional wave (P4) is due to the second fluid phase (nonwetting fluid) in the primary pores. The second, third and fourth compressional waves disappear when the frequency approaches zero and are associated with diffusive-type processes, i.e., highly attenuated. The rotational wave (S) is analogous to the rotational wave in elastic solids. All waves are dispersed and attenuated. Especially, the second, third and fourth compressional waves are highly attenuated. Because of the high attenuation, an experimental confirmation of these waves can be very difficult. We numerically examined the frequency, saturation, volume fraction of fractures dependence of phase velocity and attenuation coefficient of body waves. The third and fourth compressional waves do not depend on the volume fraction of fractures. The phase velocities of the first compressional and rotational waves slightly change due to the volume fraction of fractures. However, we observed a change in the order of magnitude of the attenuation coefficients of the first compressional and rotational waves due to the presence of the fractures. This can be explained by the high intrinsic permeability of the fractures. 


\section{References}

Bear, J. and Corapcioglu, M. Y.: 1989, Wave propagation in porous media-governing equations, in: D. Karamanlidis and R. B. Stout (eds), Wave Propagation in Granular Media, ASME, New York, pp. 91-94.

Bedford, A. and Stern, M. A.: 1983, A model for wave propagation in gassy sediments, J. Acoust. Soc. Am. 73, 409-417.

Beskos, D. E.: 1989, Dynamics of saturated rocks, I: Equations of motion, J. Eng. Mech. ASCE 115, 983-995.

Beskos, D. E., Vgenopoulou, I. and Providakis, C. P.: 1989a, Dynamics of Saturated rocks II: Body forces, J. Eng. Mech. ASCE 115, 996-1016.

Beskos, D. E., Papadakis, C. N. and Woo, H. S.: 1989b, Dynamics of saturated rocks, III: Rayleigh waves, J. Eng. Mech. ASCE 115, 1017-1034.

Beskos, D. E. and Aifantis, E. C.: 1986, On the theory of consolidation with double porosity-II, Int. J. Eng. Sci. 24, 1697-1716.

Biot, M. A.: 1956a, Theory of propagation of elastic wave in a fluid saturated porous solid, I. Low frequency range, J. Acoust. Soc. Am. 28, 168-178.

Biot, M. A.: 1956b, Theory of propagation elastic waves in a fluid saturated porous solid, II. Higher frequency range, J. Acoust. Soc. Am. 28, 169-191.

Brutsaert, W.: 1964, The propagation of elastic waves in unconsolidated unsaturated granular mediums, J. Geophys. Res. 69, 243-257.

Brutsaert, W. and Luthin, J. N.: 1964, The velocity of sound in soils near the surface as a function of the moisture content, J. Geophys, Res. 69, 643-652.

Corapcioglu, M. Y.: 1991, Wave propagation in porous media - A review, in: J. Bear and M. Y. Corapcioglu (eds), Transport Processes in Porous Media, Martinus Nijhoff, Dordrecht, 373-471.

Garg, S. K. and Nayfeh, A. H.: 1986, Compressional wave propagation in liquid and/or gas saturated elastic porous media, J. Appl. Phys. 60, 3045-3055.

Khaled, M. Y., Beskos, D. E. and Aifantis, E. C.: 1984, On the porosity of consolidation with double porosity - III A finite element formulation, Int. J. Num. Meth. Eng. 8, 101-123.

Nakoryakov, V. E., Kuznetsov, V. V. and Dontsov, V. E.: 1989, Pressure waves in saturated porous media, Int. J. Multiphase Flow 15, 857-875.

Plona, T. J.: 1980, Observation of a second bulk compressional wave in a porous medium at ultrasonic frequencies, Appl. Phys. Lett. 36, 259-261.

Santos, J. E., Corbero, J. M. and Douglas, J.: 1990a, Static and dynamic behaviour of a porous solid, J. Acoust. Soc. Am. 87, 1428-1438.

Santos, J. E., Douglas, J., Corbero, J. M. and Lovera, O. M.: 1990b, A model for wave propagation in a porous medium saturated by a two-phase fluid, J. Acoust. Soc. Am. 87, 1439-1448.

Tuncay, K.: 1995, Wave propagation in single- and double-porosity deformable porous media saturated by multiphase fluids, PhD Dissertation, Department of Civil Engineering, Texas A\&M University.

Tuncay, K. and Corapcioglu, M. Y.: 1996, Wave propagation in fractured porous media, Transport in Porous Media 23, 237-258.

Tuncay, K. and Corapcioglu, M. Y.: 1996a, Wave propagation in poroelastic media saturated by two fluids, submitted to J. Appl. Mech.

Wilson, R. K. and Aifantis, E. C.: 1982, On the theory of consolidation with double porosity, Int. J. Eng. Sci. 22, 1009-1035.

Wilson, R. K. and Aifantis, E. C.: 1984, A double porosity model for acoustic wave propagation in fractured-porous rock, Int. J. Eng. Sci. 22, 1209-1217.

Wyckoff, R. D. and Bosset, H. G.: 1936, The flow of gas-liquid mixture through unconsolidated sands, Physics 7, 325-345. 